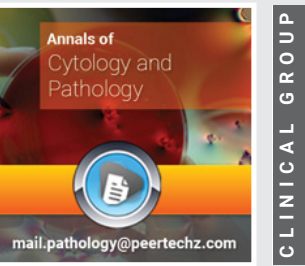

\title{
Liquid Biopsy perspectives theranostics and personalized
}

\section{oncology}

\author{
C Daniel Ascencio González* \\ Hospital Ángeles del Pedregal, Camino a Santa Teresa 1055-615- Col Héroes de Padierna, Mayor's \\ Office Magdalena Contreras C.P.10800 Mexico City, Mexico
}

Received: 15 August, 2020

Accepted: 12 September, 2020

Published: 14 September, 2020

*Corresponding author: C Daniel Ascencio González, Hospital Ángeles del Pedregal, Camino a Santa Teresa 1055-615- Col Héroes de Padierna, Mayor's Office Magdalena Contreras C.P.10800 Mexico City, Mexico, Tel: 55556858 76; E-mail: danielascencio@gmail.com

Keywords: Cancer Diagnosis; Liquid biopsy; Circulating Tumor Cells (CTCs); Circulating free Or Tumor DNA (Cfdna Or Ctdna); Extracellular Vesicles (Evs); TumorEducated platelets (Teps)

https://www. peertechz.com

Check for updates

\begin{abstract}
The current limitations of cancer diagnosis and molecular profiling based on invasive tissue biopsies or clinical imaging have led to the development of the Liquid Biopsy field (LB), includes the isolation of Circulating Tumor Cells (CTCs), circulating free or tumor DNA (cfDNA or ctDNA), Extracellular Vesicles (EVs), and TumorEducated Platelets (TEPs) from body fluid samples and their molecular characterization to identify biomarkers for early cancer diagnosis, prognosis, therapeutic prediction, and follow-up. As cancers grow, evolve, and spread, they shed circulating tumor cells (CTCs), as well as other tumor-associated cells and products, into the bloodstream. Capturing and analyzing CTCs or other tumor-associated cells and products from a patient's blood sample can provide insight into particular cancer's biology, response to treatment, and/ or potential therapeutic targets. CTCs are heterogeneous; a pressing question concerns which CTCs represent those directly involved in metastasis, the major cause of cancer-related death. The aim of this review, is to describe the biological principles underlying the Liquid Biopsy (LB) concept and to discuss how functional studies can expand the clinical applications of these circulating biomarkers.
\end{abstract}

\section{Introduction}

GLOBOCAN 2018 estimates that the incidence and mortality from cancer worldwide were 18.1 million and 9.6 million deaths, respectively is responsible for one in eight deaths worldwide. It encompasses more than 100 distinct diseases with diverse risk factors and epidemiology which originate from most of the cell types and organs of the human body and which are characterized by unrestrained proliferation of cells that can invade beyond normal tissue boundaries and metastasize to distant organs [1]. There is great potential for genome sequencing to enhance patient care through improved diagnostic sensitivity and more precise therapeutic targeting. To maximize this potential, genomics strategies that have been developed for genetic discovery - including DNA-sequencing technologies and analysis algorithms - need to be adapted to fit clinical needs. The hallmarks of cancer comprise six biological capabilities acquired during the multistep development of tumors, constitute an organizing principle for rationalizing the complexities of neoplastic disease. They include sustaining proliferative signaling, evading growth suppressors, resisting cell death, enabling replicative immortality, inducing angiogenesis, and activating invasion and metastasis [2].
All cancers arise as a result of changes that have occurred in the DNA sequence of the genomes of cancer cells, cancer development is based on two constituent processes, the continuous acquisition of heritable genetic variation in individual cells by more-or-less random mutation and natural selection acting on the resultant phenotypic diversity. Now, it will be possible to obtain the complete DNA sequence of large numbers of cancer genomes. These studies will provide us with a detailed and comprehensive perspective on how individual cancers have developed [3].

The identification of the variations of the tumor genotype demonstrate the heterogeneity parameter that predicts the therapeutic response, subpopulations of Cancer Cells (CC), unique genomes can exist within the same tumor and evolve over time (intratumor heterogeneity), which is used for characterization, monitoring of clonal dynamics and identification of therapeutic resistances [4].

\section{The Biological basis of liquid biopsies}

ctDNA and CTCs are the currently approved diagnostic biomarkers for screening cancer patients. In addition, tumorderived extracellular vesicles (tdEVs), circulating tumor- 
derived proteins, circulating tumor RNA (ctRNA) and platelets formed by tumors (TEPs) are other components of LB with diagnostic potential. The analysis of tumor cells or tumor cell products obtained from blood or other body fluids ("LB") provides a broad range of opportunities in the field of oncology. Clinical application areas include early detection of cancer or tumor recurrence, individual risk assessment and therapy monitoring. Allows to portray the entire disease as tumor cells or tumor cell products are released from all metastatic or primary tumor sites, providing comprehensive and real-time information on tumor cell evolution, therapeutic targets and mechanisms of resistance to therapy.

ctDNA fragments are released by tumor cells into the bloodstream and in principle contain genetic defects identical to the tumor cells they originate from. Accordingly, molecular alterations, which can be detected in cfDNA of plasma, span the types of genomic alterations identified in tumors and include point mutations, rearrangements, amplifications, and even gene copy variations Although fragmented, free DNA is stable in the circulation. On the contrary, free RNA molecules do not generally survive in the bloodstream. The exceptions are cellfree microRNAs that can be detected in blood plasma or serum of cancer patients. Relevant molecular information may also be obtained by analyzing RNA molecules present in extracellular vesicles such as exosomes (both coding and non-coding) or in platelets. The nucleic-acid- and protein-rich content of these nanoparticles, floating in virtually all bodily fluids, has potential for non-invasive molecular diagnostics and may represent a novel therapeutic delivery system [5].

\section{Tumor diagnosis}

Tissue biopsies are laborious, and stressful for the patient, generally unlikely to be repeated during disease progression. For these reasons, the majority of clinical decisions in the metastatic context tend to be based on biopsies of the primary cancer, and frequently do not represent the genetic profile that allows establishing the treatment of the disease, particularly of metastases [6]. The current diagnosis of tumors is studied with a tissue biopsy, it is considered the gold standard, however, it has many limitations to have an accurate diagnosis, it determines its origin and genetic profile, although it only allows studying a static and limited sample and eventually It is difficult to obtain, it has low sensitivity and precision, it does not allow determining heterogeneity or invasiveness, it is incompatible with longitudinal clinical follow-up, and it does not detect an early-stage tumor or residual tumors [7]. Specifically it does not offer an accurate diagnosis.

In oncology, detection of cfDNA derived from tumors, also known as ctDNA, has been challenging for three primary reasons, which include: discrimination of ctDNA from normal cfDNA; presence of sometimes extremely low levels of ctDNA; and the accurate quantification of the number of mutant fragments in a sample. These somatic mutations, commonly single base-pair substitutions, are present only in the genomes of cancer cells or precancerous cells and are not present in the DNA of normal cells of the same individual. This juxtaposition assures ctDNA exquisite biologic specificity as a biomarker.
The coexistence of cells of different origins and populations with different genomic and epigenomic profiles, represent the tumor genetic perspective that determines the molecular profile that allows monitoring, detecting resistance to therapy, this knowledge gives rise to the new paradigm of "Oncology, Precision", where clinical, anatomopathological, molecular, genomic, proteomic and metabolomic data are integrated ("Theranostics"), which aims to apply more precise and effective personalized treatments according to the genetic profile of each tumor [8].

The US National Cancer Institute, defines the LB as "a blood test, which studies the free nucleic acids of CTC, DNA fragments of tumor cells", represents is a complementary alternative to detect genomic alterations, predicts early relapse and response to treatment, using rapid sampling non-invasive and repeatable, it allows to dynamically identify tumor genomes in body fluids such as blood, saliva, stool, urine, pleural, cerebrospinal and ascitic fluids, allows early screening, evaluates tumor progress, therapeutic response and detects recurrences through analysis of ctDNA, CTC, which are the only components for clinical application approved by the FDA, EV, ctRNA and TEP members of the tumor circuloma are biomarkers still under study [9-11].

\section{Liquid Biopsy (LB)}

Tissue biopsies are generally time consuming, and are generally unlikely to be repeated in each cycle of disease progression. For these reasons, most clinical decisions in the metastatic setting are based on past biopsies (of the primary tumor), which generally represent the genetic makeup relevant to the treatment of the disease in its metastatic form. The reliability of tissue biopsies understanding the cancer genotype depends on several factors, such as the intrinsic dynamics of tumor evolution and the extrinsic selection pressure that results from any cancer treatment, as well as the timing and location of the tumor. biopsy itself. This has favored the appearance of the LB. Comprising analysis of circulating tumor-derived material (the 'tumor circulome'), represent an innovative tool in precision oncology to overcome current limitations associated with tissue biopsies. Within the tumor circulome, ctDNA and CTCs or circulating tumour-derived factors, in particular, ctDNA, are the only components the clinical application of which is approved diagnostic biomarkers for screening cancer patients, by the US Food and Drug Administration (FDA) are the currently approved. EVs, ctRNA, and TEPs are relatively new tumor circulome constituents with promising potential at each stage of cancer management [12]. Is a convenient fast, non-invasive and reproducible sampling method that can dynamically reflect the changes in tumor gene expression profile, and provide a robust basis for individualized therapy and early diagnosis of cancer [13].

Minimal Residual Disease (MRD) can be defined as cancer persisting in a patient after treatment that cannot be detected with current medical imaging modalities and is, therefore, an occult stage of cancer progression. LB approaches based on the detection of small numbers of CTCs or minute amounts of ctDNA now enable MRD detection in patients with various 
malignancies. CTC detection at primary diagnosis of cancer predicts an unfavourable prognosis and is, therefore, applicable to risk stratification strategies beyond the current approaches to tumour staging. Highly sensitive LB assays have been developed that can now be applied to detect and characterize MRD, which reflects the presence of tumour cells disseminated from the primary lesion to distant organs in patients who lack any clinical or radiological signs of metastasis or residual tumour cells left behind after local therapy that eventually lead to local recurrence. This application is the new frontier of LB analyses, which are challenged by the very low concentrations of CTCs and ctDNA in blood samples. Monitoring of CTCs and ctDNA during post-surgical follow-up assessments can enable the detection of disease relapse many months earlier than is possible with current radiological imaging procedures. Further characterization of CTCs and ctDNA can provide insights into the molecular evolution of MRD during tumour progression, with implications for therapy to delay or even prevent metastatic relapse [14].

Applications of Liquid Biopsy: Early detection. Assessment of molecular heterogeneity of overall disease, Monitoring of tumor dynamics, Identification of genetic determinants for targeted therapy, Evaluation of early treatment response Monitoring of minimal residual disease, Assessment of evolution of resistance in real time

\section{Latest advances and implications for therapies}

ctDNA may have utility at almost every stage of the management of patients with cancer, including diagnosis, minimally invasive molecular profiling, treatment monitoring, the detection of residual disease and the identification of resistance mutations. ctDNA analysis may be broadly considered as a tool both for quantitative analysis of disease burden and for genomic analysis. The identification of ctDNA in individuals before a cancer diagnosis, and in presymptomatic individuals, suggests the possibility of ctDNA analysis as a tool for earlier detection and screening. Noninvasive cancer classification or subtyping has also emerged as a possibility, although for early detection, both technical and biological factors introduce challenges to the detection of mutant DNA in plasma and its interpretation. Monitoring multiple mutations in parallel can enhance the sensitivity of ctDNA detection, can be used to assess the clonal evolution of patients' disease and may identify resistance mutations before clinical progression is observed. Potential applications of ctDNA have been demonstrated by a number of proof-of-principle studies. Prospective clinical trials are beginning to assess the clinical utility of ctDNA analysis for molecular profiling and disease monitoring. The increasing acceptance of ctDNA is enabling the field to move from exploratory ctDNA studies towards clinical trials in which ctDNA guides decision-making $[15,16]$.

Improvements in genomic and molecular methods are expanding the range of potential applications for ctDNA, both in a research setting and as a 'LB' for cancer management. cfDNA is released predominantly by cell death into the bloodstream, although active secretion may have a role. in the plasma of cancer patients constitutes a potential source of tumor-derived
DNA. Massively parallel sequencing analysis of cfDNA samples from cancer patients revealed that ctDNA accounts for only a fraction of the total cfDNA, and this fraction varies according to disease burden, site, and tumor biologic features including histology, vascularization, proliferation and apoptosis rates. ctDNA fraction is extremely low in many early-stage and some metastatic cancers. Accurate identification of tumorderived somatic variants in cfDNA requires understanding of the various biological compartments contributing to the cfDNA pool. The assay displayed high sensitivity and specificity, allowing for de novo detection of tumor-derived mutations and inference of tumor mutational burden, microsatellite instability, mutational signatures and sources of somatic mutations identified in cfDNA [17].

The degree of tumor heterogeneity in individual patients is an underinvestigated mechanism of resistance, which can only be targeted by combinatorial therapies. Addressing the challenge of low CTC numbers, apheresis can highly increase the number of CTCs, allowing better downstream analysis of intrapatient heterogeneity within research studies on selected patients. Apheresis facilitated the capture of large numbers of CTCs noninvasively with minimal morbidity and allowed the deconvolution of intrapatient heterogeneity and clonal evolution [18]. The study of EVs has the potential to identify unknown cellular and molecular mechanisms in intercellular communication and in organ homeostasis and disease. Exosomes, with an average diameter of $\sim 100$ nanometers, are a subset of EVs. The biogenesis of exosomes involves their origin in endosomes, and subsequent interactions with other intracellular vesicles and organelles generate the final content of the exosomes. Their diverse constituents include nucleic acids, proteins, lipids, amino acids, and metabolites, which can reflect their cell of origin. Exosomes are associated with immune responses, viral pathogenicity, pregnancy, cardiovascular diseases, central nervous system-related diseases, and cancer progression. The efficient exchange of cellular components through exosomes can inform their applied use in designing exosome-based therapeutics. Owing to their high abundance and stability, circulating ncRNAs have potential utility as noninvasive, blood-based biomarkers, the roles of ncRNAs in cellto-cell communication, with several ncRNAs having the ability to regulate gene expression outside the cell of origin, through EVs -mediated transfer to recipient cells, with implications for cancer progression and resistance to therapy, thus "foreign" microRNAs (miRNAs) encoded by non-human genomes (socalled xenomiroRNAs), like viral miRNAs, are present in human body fluids and can be used as biomarkers [19].

The potential of tumor-educated blood platelets (TEPs) as non-invasive biomarkers for RNA biomarker panels has been discovered. PETs are involved in the progression and spread of various solid tumors, and spliced PET RNA surrogate signatures can provide specific information on the presence, location, and molecular characteristics of cancers. Until now, PET samples from patients with different types of tumors, including lung, brain, and breast cancers, it has been shown that PETs from cancer patients are different from those with inflammatory and other non-cancerous diseases. TEP RNA may complement 
currently used biosources and biomolecules employed for liquid biopsy diagnosis, potentially enhancing the detection of cancer in an early stage and facilitating noninvasive disease monitoring $[20,21]$.

Composite biomarker panels need to be tested in clinical studies with well-established endpoints to demonstrate clinical validity and utility, which will be key to introduce LB into clinical practice. Furthermore, experimental studies must gain more knowledge on the biology of LB markers, which can then in turn be retranslated to the bedside to improve the clinical use of LB analytes.

Molecular analysis of cCTCs at single-cell resolution offers great promise for cancer diagnostics and therapeutics from simple LB. Recent development of massively parallel singlecell RNA-sequencing (scRNA-seq) provides a powerful method to resolve the cellular heterogeneity from gene expression and pathway regulation analysis. Next-generation sequencing (NGS)based methods include targeted as well as untargeted approaches and stand out due to their ability of massive parallel sequencing of millions of DNA sequences. Targeted NGS methods such as TAm-Seq, Safe-SeqS and CAPP-Seq enable to detect multiple rare mutations in ctDNA simultaneously. Even though targeted approaches show high analytical sensitivity, they are limited to mutations in a set of predefined genes, whereas untargeted approaches like whole genome sequencing or whole-exome sequencing provide the opportunity to detect novel, clinically relevant genomic aberrations without requiring information about the primary tumor. Nevertheless, clinical use of untargeted approaches is usually hampered due to lower sensitivity, higher input sample volume requirement and higher costs $[22,23]$.

\section{Conclusion future directions}

LB comprising the noninvasive analysis of circulating tumor-derived material (the 'tumor circulome'), represent an innovative tool in precision oncology to overcome current limitations associated with tissue biopsies. Within the ctDNA) and CTCs are the only components the clinical application of which is approved by the US Food and Drug Administration (FDA). EVs, ctRNA, and TEPs are relatively new tumor circulome constituents with promising potential at each stage of cancer management. Up to now, ctDNA has shown many promising results for cancer classification, monitoring, prognosis, and treatment selection. However, using ctDNA for cancer screening and early detection remained to be solved. The biggest challenge is the low concentration of ctDNA in the blood. Although some NGS-based protocols improve the sensitivity of ctDNA assays in many different ways, the tradeoff between sensitivity and cost is still the greatest concern in practice. In the future, other sources of information apart from ctDNA should be combined to increase sensitivity and specificity. Moreover, applying ctDNA sequencing to cancer screening provides us with a good opportunity to collect longitudinal data to create a better disease classification model. Provide a cost-effective, fast, reproducible and non-invasive source for early cancer diagnosis and prognostic monitoring. In addition, analysis of circulating tumor-derived factors or the tumor circulome in the liquid biopsies can capture the clonal heterogeneity of these tumors unlike tissue biopsies. Various LB samples can be combined to improve the chances of cancer diagnosis, and sequential real-time biopsies will further aid in the early identification of therapy-resistant tumors. Furthermore, detection and characterization of minimal residual disease after initial therapy can also be improved by analyzing LB.

\section{References}

1. Bray F, Ferlay J, Soerjomataram I, Siegel RL, Torre LA, et al. (2018) Global cancer statistics 2018: GLOBOCAN estimates of incidence and mortality worldwide for 36 cancers in 185 countries. CA Cancer J Clin 68: 394-424. Link: https://bit.ly/2Zs2nB7

2. Hanahan D, Weinberg RA (2011) Hallmarks of cancer: the next generation. Cell 144: 646-674. Link: https://bit.ly/32lxVdX

3. Stratton MR (2009) The cancer genome. Nature 7239: 719-724. Link: https://go.nature.com/3iq6tRS

4. McGranahan N, Swanton C (2017) Clonal Heterogeneity and Tumor Evolution: Past, Present, and the Future. Cell 168: 613-628. Link: https://bit.ly/3iq6xkA

5. Bardelli A, Pantel K (2017) Liquid Biopsies, What We Do Not Know (Yet). Cancer Cell 31: 172-179. Link: https://bit.ly/2GUngyN

6. Heidrich I, Ačkar L, Mossahebi Mohammadi P, Pantel K (2020) Liquid biopsies: Potential and challenges. Int J Cancer. Link: https://bit.ly/3hpH8pH

7. Thierry AR, El Messaoudi S, Gahan PB, Anker P, Stroun M (2016) Origins, structures, and functions of circulating DNA in oncology. Cancer Metastasis Rev 35: 347-376. Link: https://bit.ly/2FdqHjA

8. Takeuchi S, Okuda S (2019) Knowledge base toward understanding actionable alterations and realizing precision oncology. Int J Clin Oncol 24: 123-130. Link: https://bit.ly/32hA2Q2

9. Chu D, Park BH (2017) Liquid biopsy: unlocking the potentials of cell-free DNA Virchows Arch 471: 147-154. Link: https://bit.ly/32luRyg

10. Jung A, Kirchner T (2018) Liquid Biopsy in Tumor Genetic Diagnosis. Dtsch Arztebl Int 115: 169-174. Link: https://bit.ly/2ZrJxKy

11. Alix-Panabières C, Pantel K (2016) Clinical Applications of Circulating Tumor Cells and Circulating Tumor DNA as Liquid Biopsy. Cancer Discov 6: 479-491. Link: https://bit.ly/3bLSdjX

12. De Rubis G, Rajeev Krishnan S, Bebawy M (2019) Liquid Biopsies in Cancer Diagnosis, Monitoring, and Prognosis. Trends Pharmacol Sci 40: 172-186. Link: https://bit.ly/2GIANZU

13. Wu J, Hu S, Zhang L, Xin J, Sun C, et al. (2020) Tumor circulome in the liquid biopsies for cancer diagnosis and prognosis. Theranostics 10: 4544-4556. Link: https://bit.ly/2DPITjw

14. Pantel K, Alix-Panabières (2019) Liquid biopsy and minimal residual disease - latest advances and implications for cure. Nat Rev Clin Oncol 16: 409-424. Link: https://go.nature.com/3hkwfWp

15. Diamantopoulou Z, Castro-Giner F, Aceto N (2020) Circulating tumo cells: Ready for translation?. J Exp Med 217: e20200356. Link: https://bit.ly/32hAQo2

16. Wan JCM, Massie C, Garcia-Corbacho J, Mouliere F, Brenton JD, et al. (2017) Liquid biopsies come of age: towards implementation of circulating tumour DNA. Nat Rev Cancer 17: 223-238. Link: https://bit.ly/3hplalz

17. Razavi P, Li BT, Brown DN, Jung B, Hubbell $E$, et al. (2019) High-intensity sequencing reveals the sources of plasma circulating cell-free DNA variants. Nat Med 25: 1928-1937. Link: https://bit.ly/3mbdIPY

Citation: Ascencio González CD (2020) Liquid Biopsy perspectives theranostics and personalized oncology. Ann Cytol Pathol 5(1): 073-077. 
18. Lambros MB, Seed G, Sumanasuriya S, Gil V, Crespo M, et al. (2018) Single-cell analyses of prostate cancer liquid biopsies acquired by apheresis. Clin Cancer Res 24: 5635- 5644. Link: https://bit.ly/2DQ7SSN

19. Kalluri R, LeBleu VS (2020) The biology, function, and biomedical applications of exosomes. Science 367: eaau6977. Link: https://bit.ly/3irqTKs

20. Best MG, Wesseling $P$, Wurdinger $T$ (2018) Tumor-educated platelets as a noninvasive biomarker source for cancer detection and progression monitoring. Cancer Res 78: 3407-3412. Link: https://bit.ly/2FndnZI
21. Anfossi S, Babayan A, Pantel K, Calin GA (2018) Clinical utility of circulating non-coding RNAs-an update. Nat Rev Clin Oncol 15: 541- 563. Link: https://go.nature.com/2Fn59B1

22. Cheng YH, Chen YC, Lin E, Brien R, Jung S, et al. (2019) Hydro-Seq enables contamination-free high-throughput single-cell RNA-sequencing for circulating tumor cells. Nat Commun 10: 2163. Link: https://bit.ly/3k1gEg2

23. Chen M, Zhao H (2019) Next-generation sequencing in liquid biopsy: cancer screening and early detection. Hum Genomics 13: 34 . Link: https://bit.ly/3bZsqVH

\section{Discover a bigger Impact and Visibility of your article publication with} Peertechz Publications

\section{Highlights}

* Signatory publisher of ORCID

- Signatory Publisher of DORA (San Francisco Declaration on Research Assessment)

* Articles archived in worlds' renowned service providers such as Portico, CNKI, AGRIS, TDNet, Base (Bielefeld University Library), CrossRef, Scilit, J-Gate etc.

* Journals indexed in ICMJE, SHERPA/ROMEO, Google Scholar etc.

* OAI-PMH (Open Archives Initiative Protocol for Metadata Harvesting)

* Dedicated Editorial Board for every journa

* Accurate and rapid peer-review process

- Increased citations of published articles through promotions

* Reduced timeline for article publication

Submit your articles and experience a new surge in publication services (https://www.peertechz.com/submission).

Peertechz journals wishes everlasting success in your every endeavours.

Copyright: (C) 2020 Ascencio González CD. This is an open-access article distributed under the terms of the Creative Commons Attribution License, which permits unrestricted use, distribution, and reproduction in any medium, provided the original author and source are credited

Citation: Ascencio González CD (2020) Liquid Biopsy perspectives theranostics and personalized oncology. Ann Cytol Pathol 5(1): 073-077. 